\title{
One-Sided Cumulative Sum (CUSUM) Control Charts for the Erlang-Truncated Exponential Distribution
}

\author{
G. Srinivasa Rao \\ Department of Statistics, School of Mathematical Sciences, University of Dodoma \\ Dodoma, Tanzania, PO Box:259 \\ E-mail: gaddesrao@yahoo.com
}

Received: 13 September 2013; revised: 15 October 2013; accepted: 17 October 2013; published online: 10 December 2013

\begin{abstract}
In this article, we construct one-sided cumulative sum (CUSUM) control charts for controlling the parameters of a random variable with erlang-truncated exponential distribution. The rejection of the Wald's sequential probability ratio test (SPRT) is viewed as the decision lines of a CUSUM control chart for which the variate is a quality characteristic. Parameters of the CUSUM chart, e.g. lead distance and mask angle, are presented. The results show that the Average Run Length (ARL) of the resulting control charts changes substantially for a slight shift in the parameters of the distribution.

Key words: Sequential Probability Ratio Test (SPRT), Cumulative Sum (CUSUM) Control Chart, Average Run Length (ARL), erlang-truncated exponential distribution
\end{abstract}

\section{INTRODUCTION}

In statistical quality control the cumulative sum control charts (CUSUM Charts) have found importance as a parallel process control technique to the well-known Shewhart control charts. An alternative method for testing statistical hypothesis parallel to Neyman's theory is the popular sequential probability ratio test (SPRT) due to Wald (1942). Page (1954, 1961) suggested the cumulative sum charts which are more effective than Shewhart control charts in detecting small and moderate size departures from a simple acceptable quality level [Montgomery (2001)]. In this article we develop onesided CUSUM control charts for erlang-truncated exponential distribution to detect the shift of the process parameters. We have also examined how the parameters of the V-mask are influenced by the probability of defectives departure from its target value and the average run length (ARL) of the CUSUM scheme. Johnson (1961) introduced this method of construction of the CUSUM chart. Johnson and Leone (1962) made use of simultaneous applications of SPRT to test a simple $H_{0}$ against two separate simple alternative hypotheses on either side of the null hypothesis. The concerned decision lines for both cases of alternative hypotheses to come to the respec- tive rejection of the null hypothesis are taken to construct a cumulative sum control chart for a process variate assumed to follow a normal distribution. Johnson (1966) extended the same procedure to a CUSUM chart for the Weibull process variate. Nabar and Bilgi (1994) extended the CUSUM chart procedure to the case of inverse Gaussian distribution. Kantam and Rao (2006) studied the cumulative sum control chart for log-logistic distribution. Chakraborty and Khurshid (2011) constructed one-sided cumulative sum (CUSUM) control charts the zero-truncated binomial distribution.

The rest of the paper is organized as follows: the CUSUM chart for control of parameter $\lambda$ when $\nu$ is known along with ARL is given in Section 2. The CUSUM chart for control of parameter $\nu$ when $\lambda$ is known along with ARL is shown in Section 3, and conclusions are listed in Section 4.

\section{THE CUSUM CHART FOR CONTROL OF PARAMETER $\lambda$ WHEN $\nu$ IS KNOWN}

The Erlang-truncated exponential (ETE) distribution was introduced and studied by El-Alosey (2007), Mohsin (2009) and Mohsin et al (2010). The erlang-truncated exponential 
distribution has the following density function

$f(x ; \nu, \lambda)=\nu\left(1-e^{-\lambda}\right) \exp \left[-\nu x\left(1-e^{-\lambda}\right)\right] ; \quad$ for $x>0$

and the distribution function

$$
F(x ; \nu, \lambda)=1-\exp \left[-\nu x\left(1-e^{-\lambda}\right)\right] ; \quad \text { for } x>0 .
$$

Here $\nu>0$ and $\lambda>0$ are the shape and scale parameters, respectively. Now onwards ETE distribution with the shape parameter $\nu$ and scale parameter $\lambda$ will be denoted by $\operatorname{ETE}(\nu, \lambda)$.

The mean and variance of ETE $(\nu, \lambda)$ are $\left[\nu\left(1-e^{-\lambda}\right)\right]^{-1}$ and $\left[\nu\left(1-e^{-\lambda}\right)\right]^{-2}$, respectively.

If we assume that $X_{1}, X_{2}, \ldots X_{m}$ be i.i.d. random variables taken from ETE distribution with the probability density function (1). The likelihood ratio to test the null hypothesis $H_{0}: \lambda=\lambda_{0}$ against the alternative hypothesis $H_{1}: \lambda=$ $\lambda_{1}\left(>\lambda_{0}\right)$ with known $\nu$ is given by

$$
\begin{aligned}
& \frac{L_{1}}{L_{0}}=\frac{f_{X_{1}, X_{2}, \ldots X_{m}}\left(x_{1}, x_{2}, \ldots x_{m} ; \lambda_{1}, \nu\right)}{f_{X_{1}, X_{2}, \ldots X_{m}}\left(x_{1}, x_{2}, \ldots x_{m} ; \lambda_{0}, \nu\right)}= \\
= & \left(\frac{1-e^{-\lambda_{1}}}{1-e^{-\lambda_{0}}}\right)^{m} \exp \left[\nu\left(e^{-\lambda_{1}}-e^{-\lambda_{0}}\right) \sum_{i=1}^{m} x_{i}\right]
\end{aligned}
$$

The continuation region of the sequential probability ratio test (SPRT) discriminating between the two hypotheses is given by

$$
\begin{gathered}
\ln \left(\frac{\beta}{1-\alpha}\right)<m \ln \left(\frac{1-e^{-\lambda_{1}}}{1-e^{-\lambda_{0}}}\right) \\
+\nu\left(e^{-\lambda_{1}}-e^{-\lambda_{0}}\right) \sum_{i=1}^{m} x_{i}<\ln \left(\frac{1-\beta}{\alpha}\right)
\end{gathered}
$$

where $\alpha$ and $\beta$ are the probability of Type I and Type II errors, respectively.

If $\beta=0$ then the right side of inequality in (4) becomes

$$
\sum_{i=1}^{m} x_{i}<\frac{\ln \alpha+m \ln \left(\frac{1-e^{-\lambda_{1}}}{1-e^{-\lambda_{0}}}\right)}{\nu\left(e^{-\lambda_{0}}-e^{-\lambda_{1}}\right)}
$$

The cumulative sum (CUSUM) control chart (as shown in Fig. 1) is constructed as follows: The CUSUM control chart is formed by plotting the sum $S_{m}=\sum_{i=1}^{m} x_{i}$ against the number of observations $m$. A visual procedure with aid of the V-Mask is sometimes used to determine whether the process is under control or out of control (see Fig. 1). A V-Mask is an overlay shape in the form of a V on its side that is superimposed on the graph of the cumulative sums. The origin point of the V-Mask (see Fig. 1) is placed on top of the latest cumulative sum point and past points are examined to see if there was any fall above or below the sides of the V. As long as all the previous points lie between the sides of the $\mathrm{V}$, the process is in control. Otherwise (even if one point lies outside) the process is suspected of being out of control. Suppose here $\mathrm{O}$ is the last plotted point, $\mathrm{P}$ is the vertex of the mask and the point $\mathrm{Q}$ is obtained by drawing a perpendicular to the line OP. A small shift in the value of $\lambda$ from $\lambda_{0}$ to $\lambda_{1}$ is indicated if any plotted point falls below the line PQ. Lucas (1982) proposed a control chart which consists of control lines rather than a V-mask, in our study; we prefer to use the original $\mathrm{V}$-mask procedure.

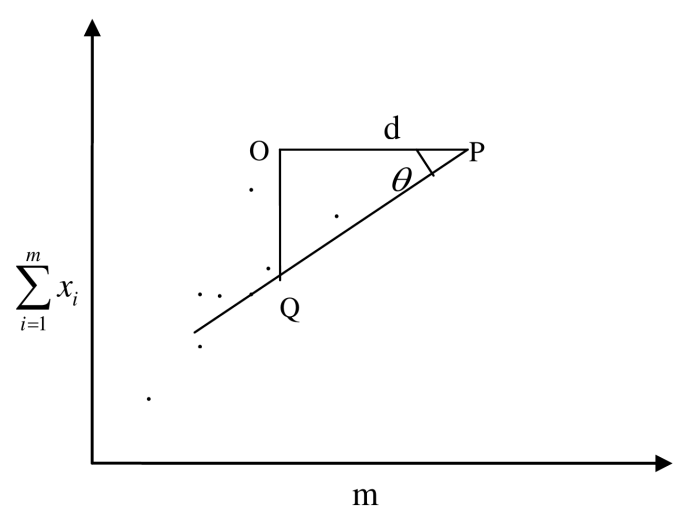

Fig. 1. Cumulative sum control chart

The parameters $d$ and $\theta$ of V-mask from Fig. 1 are given by

$$
d=\frac{-\ln \alpha}{\ln \left(\frac{1-e^{-\lambda_{1}}}{1-e^{-\lambda_{0}}}\right)}
$$

and

$$
\theta=\tan ^{-1}\left[\frac{\ln \left(\frac{1-e^{-\lambda_{1}}}{1-e^{-\lambda_{0}}}\right)}{\nu\left(e^{-\lambda_{0}}-e^{-\lambda_{1}}\right)}\right]
$$

\section{1. Average Run Length (ARL)}

In general, the average number of trials required for detecting a shift in the process average for the first time is called the average run length (ARL). If $\alpha$ is the producer's risk then the approximate formula for ARL for the CUSUM control chart [see also Johnson (1961), Johnson and Leone (1962)] detecting a shift of the parameter from $\lambda_{0}$ to $\lambda_{1}$ is given by

$$
\mathrm{ARL}=\frac{-\log \alpha}{E(\log Z)_{\lambda=\lambda_{1}}},
$$

where $Z=\frac{f\left(x ; \lambda_{1}\right)}{f\left(x ; \lambda_{0}\right)}$. Thus, we get

$$
\mathrm{ARL}=\frac{-\log \alpha}{\ln \left(\frac{1-e^{-\lambda_{1}}}{1-e^{-\lambda_{0}}}\right)-\left(\frac{e^{-\lambda_{0}}-e^{-\lambda_{1}}}{1-e^{-\lambda_{1}}}\right)}
$$

The parameters of the V-mask of the one-sided CUSUM chart, such as the lead distance $d$ and the angle $\theta$, are calculated 
for a number of combinations of the values of $\lambda, \nu$ and $\alpha$ for controlling parameters $\lambda$ when $\nu$ is known. The obtained values of $d, \theta$ and ARL for controlling parameter $\lambda$ when $\nu$ is known are displayed in the Tab. 1, 2 and 3 respectively.

\section{THE CUSUM CHART FOR CONTROL OF THE PARAMETER $\nu$ WHEN $\lambda$ IS KNOWN}

The CUSUM chart to control parameter $\nu$ when $\lambda$ is known is constructed in the following procedure. The approximate likelihood ratio for detecting a shift in the value of $\nu$ from $\nu_{0}$ to $\nu_{1}\left(>\nu_{0}\right)$ is given by

$$
\begin{gathered}
\frac{L_{1}}{L_{0}}=\frac{f_{X_{1}, X_{2}, \ldots X_{m}}\left(x_{1}, x_{2}, \ldots x_{m} ; \nu_{1}, \lambda\right)}{f_{X_{1}, X_{2}, \ldots X_{m}}\left(x_{1}, x_{2}, \ldots x_{m} ; \nu_{0}, \lambda\right)}= \\
=\left(\frac{\nu_{1}}{\nu_{0}}\right)^{m} \exp \left[-\left(\nu_{1}-\nu_{0}\right)\left(1-e^{-\lambda}\right) \sum_{i=1}^{m} x_{i}\right]
\end{gathered}
$$

Proceeding like in Section 2, we get the inequality below hold true for $\nu$

$$
\sum_{i=1}^{m} x_{i}<\frac{\ln \alpha+m \ln \left(\nu_{1} / \nu_{0}\right)}{\left(\nu_{1}-\nu_{0}\right)\left(1-e^{-\lambda}\right)}
$$

The CUSUM chart is constructed in the similar manner as we explained in Section 2 for change of $\nu$ from $\nu_{0}$ to $\nu_{1}\left(>\nu_{0}\right)$. The parameters of the V-mask (the lead distance $d$ and the angle $\theta$ ) are given by

$$
d=\frac{-\ln \alpha}{\ln \left(\nu_{1} / \nu_{0}\right)}
$$

and

$$
\theta=\tan ^{-1}\left[\frac{\ln \left(\nu_{1} / \nu_{0}\right)}{\left(\nu_{1}-\nu_{0}\right)\left(1-e^{-\lambda}\right)}\right]
$$

\section{1. Average Run Length (ARL)}

The ARL for detecting a change in $\nu$ from $\nu_{0}$ to $\nu_{1}\left(>\nu_{0}\right)$ is approximately given by

$$
\mathrm{ARL}=\frac{-\log \alpha}{E(\log Z)_{\nu=\nu_{1}}},
$$

where $Z=\frac{f\left(x ; \lambda, \nu_{1}\right)}{f\left(x ; \lambda, \nu_{0}\right)}$. Thus, we get

$$
\mathrm{ARL}=\frac{-\log \alpha}{\ln \left(\nu_{1} / \nu_{0}\right)+\frac{\nu_{0}}{\nu_{1}}-1} .
$$

The parameters of the V-mask of the one-sided CUSUM chart, such as lead distance $d$ and angle $\theta$ are calculated for a number of combinations of the values of $\lambda, \nu$ and $\alpha$ for controlling the parameters $\nu$ when $\lambda$ is known. The obtained values of $\mathrm{d}, \theta$ and ARL for controlling the parameter $\nu$ when $\lambda$ is known are displayed in the Tab. 4, 5 and 6 respectively.

\section{CONCLUSIONS}

It is noticeable from Tab. 1 for all the combinations of $\lambda$ and fixed $\alpha$ that the values of $d$ are independent from shape parameter $\nu$ and decrease as the ratio $\lambda_{1} / \lambda_{0}$ increases, whereas for constant ratio $\lambda_{1} / \lambda_{0}$, the values of $d$ increases as $\alpha$ increases for controlling parameter $\lambda$. Tab. 2 indicates that angle $\theta$ of the $\mathrm{V}$-mask decreases as the ratio $\lambda_{1} / \lambda_{0}$ increases and for constant ratio $\lambda_{1} / \lambda_{0}$ the angle decreases as $\nu$ increases for controlling parameter $\lambda$.

Tab. 1. Values of $d$ for controlling parameter $\lambda$ when $\nu$ is known

\begin{tabular}{l|l|l|l|l|l|l}
\hline \multirow{2}{*}{$\lambda_{0}$} & \multirow{2}{*}{$\lambda_{1}$} & \multicolumn{5}{|c}{$\alpha$} \\
\cline { 3 - 7 } & & 0.05 & 0.025 & 0.01 & 0.005 & 0.001 \\
\hline 0.50 & 0.55 & 41.33 & 50.89 & 63.53 & 73.09 & 95.30 \\
\hline 0.50 & 0.60 & 21.89 & 26.95 & 33.64 & 38.71 & 50.47 \\
\hline 0.50 & 0.65 & 15.40 & 18.96 & 23.68 & 27.24 & 35.51 \\
\hline 0.50 & 0.70 & 12.16 & 14.97 & 18.69 & 21.50 & 28.03 \\
\hline 0.50 & 0.75 & 10.21 & 12.57 & 15.70 & 18.06 & 23.54 \\
\hline 0.50 & 0.80 & 8.91 & 10.97 & 13.70 & 15.76 & 20.55 \\
\hline 0.50 & 0.85 & 7.99 & 9.83 & 12.28 & 14.12 & 18.41 \\
\hline 0.50 & 0.90 & 7.29 & 8.98 & 11.21 & 12.89 & 16.81 \\
\hline 0.50 & 0.95 & 6.75 & 8.31 & 10.38 & 11.94 & 15.57 \\
\hline 0.50 & 1.00 & 6.32 & 7.78 & 9.71 & 11.18 & 14.57 \\
\hline
\end{tabular}

Tab. 3 depicts the values of ARL (an average number of observations required to detect the shift of the process parameter) for different combinations of $\alpha, \nu, \lambda_{0}$ and $\lambda_{1}$. Here it is interesting to note that ARL is independent of shape parameter $\nu$, similar as in the case of lead distance d. It seems to be evident from Tab. 3 that for fixed $\alpha$ and $\lambda_{0}$ the ARL decreases as $\lambda_{1}$ increases (or the ratio increases), and for fixed values $\lambda_{0}$ and $\lambda_{1}$ the ARL increases as $\alpha$ decreases for controlling parameter $\lambda$.

Tab. 4 shows that for all the combinations of $\nu$ and fixed $\alpha$, the values of $d$ are independent of scale parameter $\lambda$ and decrease as the ratio $\nu_{1} / \nu_{0}$ increases, whereas for constant ratio $\nu_{1} / \nu_{0}$, the values of $d$ increases as $\alpha$ increases for controlling parameter $\nu$. Tab. 5 indicates that angle $\theta$ of the V-mask decreases as the ratio $\nu_{1} / \nu_{0}$ increases and for constant ratio $\nu_{1} / \nu_{0}$ the angle decreases as $\lambda$ increases for controlling parameter $\nu$.

Tab. 6 describes the values of ARL (an average number of observations required to detect the shift of the process parameter) for different combinations of $\alpha, \lambda, \nu_{0}$ and $\nu_{1}$. Here also ARL is independent of scale parameter $\lambda$, similar as in the case of lead distance d. It is clearly evident from Tab. 6 that for fixed $\alpha$ and $\nu_{0}$ the ARL decreases as $\nu_{1}$ increases (or the ratio increases), and for fixed values $\nu_{0}$ and $\nu_{1}$ the ARL increases as $\alpha$ decreases for controlling parameter $\nu$.

Moreover, in comparison to angle $\theta$, the ARL and the values of $d$ for controlling $\lambda$ differ from those for controlling $\nu$. 
From Tables 1 and 4, we have observed that the values of $d$ for controlling the parameter $\lambda$, when $\nu$ is known, and we have concluded that $d$ follows the same trends as in the case of controlling $\nu$ when $\lambda$ is known but that the values of $d$ for controlling $\nu$ are smaller than for controlling $\lambda$. The similar trend follows for angle $\theta$ and also the ARL values. Among these two CUSUM control charts the values of parameters $d$, $\theta$ and ARL for controlling $\nu$ are uniformly smaller than for controlling $\lambda$.

Tab. 2. Values of $\theta$ for controlling parameter $\lambda$ when $\nu$ is known

\begin{tabular}{l|l|l|l|l|l|l|l|l|l|l}
\hline \multirow{2}{*}{$\lambda_{0}$} & \multirow{2}{*}{$\lambda_{1}$} & \multicolumn{10}{|c}{$\nu$} \\
\cline { 3 - 11 } & & 0.60 & 0.65 & 0.70 & 0.75 & 0.80 & 0.85 & 0.90 & 0.95 & 1.00 \\
\hline 0.50 & 0.55 & 76.24 & 75.14 & 74.06 & 72.98 & 71.92 & 70.87 & 69.83 & 68.81 & 67.80 \\
\hline 0.50 & 0.60 & 75.80 & 74.67 & 73.56 & 72.45 & 71.36 & 70.28 & 69.22 & 68.17 & 67.14 \\
\hline 0.50 & 0.65 & 75.39 & 74.23 & 73.09 & 71.96 & 70.84 & 69.74 & 68.65 & 67.58 & 66.52 \\
\hline 0.50 & 0.70 & 75.01 & 73.83 & 72.65 & 71.50 & 70.36 & 69.23 & 68.12 & 67.03 & 65.95 \\
\hline 0.50 & 0.75 & 74.66 & 73.45 & 72.25 & 71.07 & 69.91 & 68.76 & 67.63 & 66.52 & 65.43 \\
\hline 0.50 & 0.80 & 74.33 & 73.09 & 71.87 & 70.67 & 69.49 & 68.32 & 67.17 & 66.04 & 64.94 \\
\hline 0.50 & 0.85 & 74.01 & 72.76 & 71.52 & 70.30 & 69.10 & 67.91 & 66.75 & 65.60 & 64.48 \\
\hline 0.50 & 0.90 & 73.72 & 72.45 & 71.19 & 69.95 & 68.73 & 67.53 & 66.35 & 65.19 & 64.05 \\
\hline 0.50 & 0.95 & 73.45 & 72.16 & 70.88 & 69.62 & 68.39 & 67.17 & 65.98 & 64.80 & 63.65 \\
\hline 0.50 & 1.00 & 73.19 & 71.88 & 70.59 & 69.32 & 68.06 & 66.83 & 65.63 & 64.44 & 63.28 \\
\hline
\end{tabular}

Tab. 3. Values of ARL for controlling parameter $\lambda$ when $\nu$ is known

\begin{tabular}{l|l|l|l|l|l|l}
\hline \multirow{2}{*}{$\lambda_{0}$} & \multirow{2}{*}{$\lambda_{1}$} & \multicolumn{5}{|c}{$\alpha$} \\
\cline { 3 - 7 } & & 0.05 & 0.025 & 0.01 & 0.005 & 0.001 \\
\hline 0.50 & 0.55 & 1167.98 & 1438.22 & 1795.47 & 2065.71 & 2693.20 \\
\hline 0.50 & 0.60 & 334.53 & 411.93 & 514.25 & 591.65 & 771.38 \\
\hline 0.50 & 0.65 & 168.79 & 207.84 & 259.47 & 298.53 & 389.21 \\
\hline 0.50 & 0.70 & 106.94 & 131.69 & 164.40 & 189.14 & 246.60 \\
\hline 0.50 & 0.75 & 76.57 & 94.29 & 117.71 & 135.43 & 176.56 \\
\hline 0.50 & 0.80 & 59.13 & 72.81 & 90.90 & 104.58 & 136.35 \\
\hline 0.50 & 0.85 & 48.06 & 59.17 & 73.87 & 84.99 & 110.81 \\
\hline 0.50 & 0.90 & 40.51 & 49.88 & 62.27 & 71.64 & 93.40 \\
\hline 0.50 & 0.95 & 35.08 & 43.20 & 53.93 & 62.05 & 80.90 \\
\hline 0.50 & 1.00 & 31.03 & 38.21 & 47.70 & 54.88 & 71.56 \\
\hline
\end{tabular}

Tab. 4. Values of $d$ for controlling parameter $\nu$ when $\lambda$ is known

\begin{tabular}{l|l|l|l|l|l|l}
\hline \multirow{2}{*}{$\nu_{0}$} & \multirow{2}{*}{$\nu_{1}$} & \multicolumn{5}{|c}{$\alpha$} \\
\cline { 3 - 7 } & & 0.05 & 0.025 & 0.01 & 0.005 & 0.001 \\
\hline 0.60 & 0.65 & 37.43 & 46.09 & 57.53 & 66.19 & 86.30 \\
\hline 0.60 & 0.70 & 19.43 & 23.93 & 29.87 & 34.37 & 44.81 \\
\hline 0.60 & 0.75 & 13.43 & 16.53 & 20.64 & 23.74 & 30.96 \\
\hline 0.60 & 0.80 & 10.41 & 12.82 & 16.01 & 18.42 & 24.01 \\
\hline 0.60 & 0.85 & 8.60 & 10.59 & 13.22 & 15.21 & 19.83 \\
\hline 0.60 & 0.90 & 7.39 & 9.10 & 11.36 & 13.07 & 17.04 \\
\hline 0.60 & 0.95 & 6.52 & 8.03 & 10.02 & 11.53 & 15.03 \\
\hline 0.60 & 1.00 & 5.86 & 7.22 & 9.02 & 10.37 & 13.52 \\
\hline
\end{tabular}


Tab. 5. Values of $\theta$ for controlling parameter $\nu$ when $\lambda$ is known

\begin{tabular}{|c|c|c|c|c|c|c|c|c|c|c|c|c|}
\hline \multirow{2}{*}{$\nu_{0}$} & \multirow{2}{*}{$\nu_{1}$} & \multicolumn{11}{|c|}{$\lambda$} \\
\hline & & 0.50 & 0.55 & 0.60 & 0.65 & 0.70 & 0.75 & 0.80 & 0.85 & 0.90 & 0.95 & 1.00 \\
\hline 0.60 & 0.65 & 76.19 & 75.20 & 74.26 & 73.38 & 72.54 & 71.76 & 71.02 & 70.32 & 69.66 & 69.04 & 68.45 \\
\hline 0.60 & 0.70 & 75.68 & 74.65 & 73.69 & 72.77 & 71.91 & 71.10 & 70.34 & 69.62 & 68.94 & 68.31 & 67.70 \\
\hline 0.60 & 0.75 & 75.18 & 74.13 & 73.13 & 72.19 & 71.30 & 70.47 & 69.69 & 68.95 & 68.25 & 67.60 & 66.98 \\
\hline 0.60 & 0.80 & 74.70 & 73.61 & 72.58 & 71.62 & 70.71 & 69.86 & 69.05 & 68.29 & 67.58 & 66.91 & 66.28 \\
\hline 0.60 & 0.85 & 74.23 & 73.11 & 72.06 & 71.07 & 70.13 & 69.26 & 68.43 & 67.66 & 66.93 & 66.24 & 65.60 \\
\hline 0.60 & 0.90 & 73.77 & 72.62 & 71.54 & 70.52 & 69.57 & 68.67 & 67.83 & 67.04 & 66.29 & 65.59 & 64.93 \\
\hline 0.60 & 0.95 & 73.32 & 72.14 & 71.03 & 70.00 & 69.02 & 68.11 & 67.25 & 66.44 & 65.68 & 64.96 & 64.29 \\
\hline 0.60 & 1.00 & 72.88 & 71.67 & 70.54 & 69.48 & 68.49 & 67.55 & 66.67 & 65.85 & 65.08 & 64.35 & 63.17 \\
\hline
\end{tabular}

Tab. 6. Values of ARL for controlling parameter $\nu$ when $\lambda$ is known

\begin{tabular}{l|l|l|l|l|l|l}
\hline \multirow{2}{*}{$\nu_{0}$} & \multirow{2}{*}{$\nu_{1}$} & \multicolumn{5}{|c}{$\alpha$} \\
\cline { 3 - 7 } & & 0.05 & 0.025 & 0.01 & 0.005 & 0.001 \\
\hline 0.60 & 0.65 & 960.28 & 1182.47 & 1476.19 & 1698.38 & 2214.29 \\
\hline 0.60 & 0.70 & 265.26 & 326.64 & 407.77 & 469.15 & 611.66 \\
\hline 0.60 & 0.75 & 129.44 & 159.39 & 198.98 & 228.93 & 298.47 \\
\hline 0.60 & 0.80 & 79.50 & 97.89 & 122.21 & 140.61 & 183.32 \\
\hline 0.60 & 0.85 & 55.28 & 68.07 & 84.98 & 97.77 & 127.48 \\
\hline 0.60 & 0.90 & 41.53 & 51.14 & 63.84 & 73.45 & 95.77 \\
\hline 0.60 & 0.95 & 32.88 & 40.49 & 50.54 & 58.15 & 75.82 \\
\hline 0.60 & 1.00 & 27.03 & 33.29 & 41.55 & 47.81 & 62.33 \\
\hline
\end{tabular}

\section{References}

[1] A.B. Chakraborty, A. Khurshid, One-Sided Cumulative Sum (CUSUM) Control Charts for the Zero-Truncated Binomial Distribution, Economic Quality Control 26, 41-51 (2011).

[2] A.R. El-Alosey, Random sum of new type of mixture of distribution, International Journal of Statistics and Systems 2, 49-57 (2007).

[3] N.L. Johnson, A simple theoretical approach to Cumulative sum control charts, Journal of Amer. Statist. Assoc. 56, 835840 (1961).

[4] N. L. Johnson, Cumulative sum control charts and the Weibull distribution, Technometrics 8 (3), 481-491 (1966).

[5] N.L. Johnson, F.C. Leone, Cumulative sum control charts: Mathematical principles applied to their construction and use, Indust. Qual. Control 19, 22-28 (1962).

[6] R.R.L. Kantam, G.S. Rao, Cumulative Sum Control Chart for log-logistic distribution, InterStat, online Journal, July, 1-9 (2006).
[7] J.M. Lucas, Combined Shewhart-CUSUM quality control schemes, Journal of Quality Technology 14, 51-59 (1982).

[8] M. Mohsin, Recurrence relation for single and product moments of record values from Erlang-truncated exponential distribution, World Applied Science Journal, 6, 279-282 (2009).

[9] M. Mohsin, S. Shahbaz, M.Q. Shahbaz, A characterization of Erlang-truncated exponential distribution in record values and its use in mean residual life, Pakistan Journal of Statistics and Operations Research 6(2), 143-148 (2010).

[10] D.C. Montgomery, Introduction to Statistical Quality Control, Third edition, John Wiley\&Sons, New York 2001.

[11] S.P. Nabar, S. Bilgi, Cumulative sum control chart for the Inverse Gaussian distribution, Journal of Indian Statistical Association 32, 9-14 (1994).

[12] E.S. Page, Continuous inspection schemes, Biometrika 41, 100-115 (1954).

[13] E.S. Page, Cumulative sum charts, Technometrics 3, 1-9 (1961).

[14] A. Wald, Sequential analysis, John Wiley\&Sons, New York 1947. 


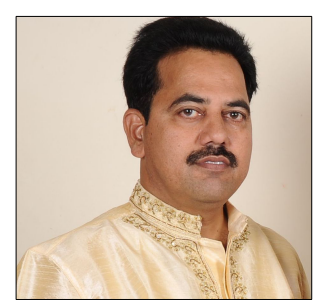

Gadde Srinivasa Rao was born on $25^{\text {th }}$ August, 1965 in Guntur, AP, India. He received the BSc (1985), MSc (1988), M.Phil. (1994) and PhD (2002) in Statistics from the Acharya Nagarjuna University, Guntur, India. He is presently working as Professor of Statistics at the Department of Statistics, University of Dodoma, Tanzania. His research interests include statistical inference, statistical process control, acceptance sampling plans and reliability estimation. He boasts more than 50 publications in different peer-reviewed journals. 